\title{
Asistente de velocidad vehicular como agente de control en entornos urbanos
}

\author{
Rodrigo Velázquez \\ Instituto Politécnico Nacional, Centro de Investigación en Computación, \\ Ciudad de México, México
}

\begin{abstract}
Resumen. En la actualidad es común encontrar vehículos cada vez más seguros y confortables, esto se debe al desarrollo tecnológico de los últimos años y a diversas iniciativas y proyectos a nivel mundial, entre las cuales podríamos citar el proyecto AutoNOMOS Labs a cargo del Dr. Raúl Rojas de la Universidad Libre de Berlín. Como ejemplo de estos avances se pueden mencionar algunos sistemas como el de antibloqueo de frenos (ABS), control crucero (CC) y, sistema asistente de velocidad inteligente (ISA). En este trabajo, primero se presentan diferentes implementaciones de asistentes de velocidad inteligente, que permiten asistir al conductor sobre la velocidad que lleva con el vehículo y, en determinadas ocasiones tomar el control de la aceleración y frenado. En una segunda etapa del trabajo se propone el modelo de un sistema asistente de velocidad, basado en coordenadas de mapas digitales y la ubicación actual del vehículo. Cuando la información de latitud y la longitud del GPS instalado en el vehículo se aproxime a las coordenadas que están almacenadas en la base de datos mariaDB (ingresadas a través de una aplicación desarrollada con JavaScript y Google Maps), verificará el límite de velocidad almacenado y la velocidad del vehículo para emitir una alerta al conductor.
\end{abstract}

Palabras clave: asistente de velocidad, mapas digitales, vehículos.

\section{Vehicle Speed Assistant as a Control Agent in Urban Environments}

\begin{abstract}
Currently, it is common to find vehicles that are increasingly safe and comfortable, this is due to the technological development of recent years and various initiatives and projects worldwide, among which we could mention the AutoNOMOS Labs project, led by Dr. Raúl Rojas at the Free University of Berlin. As an example of these advances we can mention some systems such as the anti-lock braking system (ABS), cruise control (CC) and intelligent speed assist system (ISA). In this work, we first present different implementations of intelligent speed assistants, which allow the driver to be assisted on the speed that he carries with the vehicle and, in certain occasions, to take control of the acceleration and braking. In a second stage of the work the model of a speed assistant system is proposed, based on coordinates of digital maps and the current
\end{abstract}


location of the vehicle. When the latitude and longitude information of the GPS installed in the vehicle is close to the coordinates that are stored in the mariaDB database (entered through an application developed with JavaScript and Google Maps), it will verify the speed limit stored and the speed of the vehicle to issue an alert to the driver.

Keywords: speed assistant, digital maps, vehicles.

\section{Introducción}

El presente trabajo busca plantear el modelo de un sistema asistente de velocidad vehicular que sea capaz de identificar coordenadas lanzadas por un dispositivo GPS en el vehículo y compararlas con las coordenadas almacenadas en una base de datos de marcas de velocidad de zonas urbanas, y alertar al conductor si excede en alguna de ellas a medida que vaya avanzando en su recorrido, lo que puede contribuir de gran manera al desarrollo de esta línea de investigación y a mejorar de forma notable las posibilidades de obtener en un futuro no muy lejano un automóvil que pueda ser completamente asistido por un computador.

Parte de las normativas que mencionamos están constituidas por las señales de tránsito que regulan el comportamiento vial de los automóviles, específicamente las señales de máxima velocidad, para nuestro caso, las mismas resguardan la seguridad de los conductores y peatones para determinadas zonas.

\subsection{Objetivos}

El objetivo de este trabajo será proponer el modelo que incluye software y hardware, de un sistema asistente de velocidad de un vehículo, según su ubicación en tiempo real, para posteriormente comunicar por medio de sus coordenadas, su ubicación con respecto a unas marcas de límite de velocidad y así poder trabajar la información procesada pudiendo utilizarla para alertar al conductor sobre exceso de velocidad.

En detalle se tienen los siguientes objetivos: Proponer el diseño del sistema asistente de velocidad, proponer pruebas y experimentos con prototipos robóticos que permitan identificar coordenadas en movimiento y preparar un plan de pruebas para diferentes escenarios que permitan perfeccionar el diseño.

\subsection{Motivación}

Los mapas se utilizan para dos propósitos principales: localización y navegación. La localización ayuda a situarnos comparando los puntos de referencia que se ven a nuestro alrededor con los puntos de referencia que figuran en el mapa y la navegación es la que nos guía de un lugar a otro en una ruta desconocida.

En este trabajo, el concepto de mapa abarcará solamente el propósito de localización, ya que tanto el usuario que ingresa las marcas como el sistema 
necesitarán establecer solamente las coordenadas de donde estará situada la máxima de velocidad y donde está el vehículo actualmente.

Este diseño surgió de la necesidad de contar con algún tipo de asistente, que permita al conductor alertar sobre los límites de velocidad, para cada zona en determinadas rutas, haciendo también posible alertar a los conductores acerca de los controles de velocidad bajo radar. Esto podría ser de gran utilidad para resguardar la seguridad tanto de peatones como del conductor y la posibilidad de evitar infracciones por exceso de velocidad.

\subsection{Estructura del trabajo}

Luego de los planteamientos presentados en función de los objetivos y la motivación de este trabajo, el contenido del mismo se organiza en el estado del arte y en la propuesta del diseño. Dentro de cada uno de ellos, y en sus secciones, se mencionan las estrategias de asistentes más relevantes y los trabajos de investigación previos y que han sido tomados como punto de inicio.

En la sección 2 se describe los conceptos que son necesarios conocer para que se pueda comprender los objetivos del presente trabajo y los últimos desarrollos existentes en el campo de la tecnología.

En la sección 3 se presenta el modelo para la implementación de un sistema asistente de velocidad para diferentes tipos de vehículos en zonas urbanas. Además, se presenta un listado de requerimientos necesarios para una posible implementación del mismo, como así también de una serie de ventajas y desventajas del diseño.

Finalmente en la sección 4 las conclusiones y resultados del presente trabajo, así como algunas líneas de investigación que podrían generarse a raíz de las contribuciones presentadas aquí.

\section{Estado del arte}

Enfocados en el Sistema Avanzado de Asistencia a la Conducción, o más conocido como Sistemas ADAS, presentamos los conceptos técnicos referentes a nuestros objetivos y a los proyectos de investigación; AutoNOMOS, de la Universidad Libre de Berlín, y el Programa Autopía, de la Universidad Politécnica de Madrid. Además, las nuevas aportaciones hechas para el control longitudinal de vehículos se presentan también en esta sección, las mismas son planteadas como diferentes tipos de estrategias aplicadas para conseguir dicho control.

\subsection{Control longitudinal}

Se trata acerca del comportamiento del vehículo, ante las diferentes entradas y perturbaciones que pudieran llegar a surgir con respecto al avance del mismo, en este caso el modelo será un modelo longitudinal, despreciando así las fuerzas 
y movimientos laterales del vehículo debido a que apenas afectan para el cálculo de la velocidad y movimiento longitudinal [6].

Los Sistemas de navegación inercial (INS), permiten determinar la posición del vehículo a partir de los valores de la aceleración lineal y la velocidad angular medidos por el sistema. Un INS es un sistema formado por sensores que miden aceleraciones y variaciones angulares. Integrando esta información y la obtenida de los odómetros, podemos calcular la posición actual del vehículo, a partir de su posición inicial [6].

\subsection{Sistema de aceleración electrónica (TAC)}

Throttle Actuador Control (TAC). La aceleración electrónica suprime el acoplamiento mecánico entre la mariposa y el acelerador en vehículos a gasolina, siendo sustituido por una conexión eléctrica por medio de una unidad de control, que a la vez comanda la inyección y el encendido del motor para un mejor arranque en frío. La entrada de aire se controla mediante una señal eléctrica, a medida que cambia la posición del pedal [1].

Con la instalación de sistemas de aceleración electrónica se consigue la reducción de los gases de escape, una aceleración controlada y precisa sobre todo en velocidades invariables [1].

Sensor de Posición de la Mariposa del Acelerador (TPS) La señal indica en qué posición está la mariposa del acelerador a la PCM, ganando el incremento de la potencia del motor. Contiene un potenciómetro en el eje de la mariposa que trabaja como una resistencia que varía según el movimiento del eje, se alimenta con 5 voltios, tiene una señal variable de voltaje hacia la ECU y masa [1].

Sensor de oxígeno Verifica que la dosificación aire/combustible sea exacta para el convertidor catalítico por medio de la señal eléctrica del sensor de oxígeno a la ECU, y ajustará la mezcla idónea a inyectar en la cantidad de aire que entra al colector de admisión. Este sensor consta de cuatro cables: 2 al calefactor del sensor, una señal y una masa [1].

Sensor de posición del pedal del acelerador (APP) Este sensor puede venir equipado en el propio pedal o a su vez que un cable de gobierno se envíe al mismo, de este modo el conductor ejecuta la acción sobre un resorte que moverá los potenciómetros del APP para saber las exigencias a las que se está realizando la aceleración. Los sensores APP pueden constar de dos o tres potenciómetros, si es un sensor de dos potenciómetros tienen dos señales diferentes por lo que el voltaje de un potenciómetro se amplía y la otra decrece al mover el pedal del acelerador.[1]

La ECU examina cómo se desenvuelven los potenciómetros verificando que las tensiones se encuentren dentro de los rangos precisados [1]. 


\subsection{Arduino Uno}

Es una plataforma que está constituida de un microcontrolador con reguladores de tensión, un puerto USB acoplado a un módulo adaptador USB-SERIE que permite programar el microcontrolador y ejecutar pruebas de comunicación con el propio chip [1].

Esta placa también dispone de 6 pines de entrada analógicos que transportan las señales a un convertidor analógico/digital de 10 bits. Tiene un lenguaje de programación Processing (integrado de códigos abiertos) [1].

\subsection{Sistemas inteligentes de transporte (ITS)}

Los Sistemas Inteligentes de Transporte (ITS) pueden ser definidos como la integración de tecnologías de comunicación y electrónica con el fin de mitigar los problemas de transporte terrestre. Una aplicación podría consistir en la implementación de límites de velocidad variable en la vía para la adaptación de la conducción al estado del tráfico, a las características de la vía o a las condiciones meteorológicas en cada momento (Gestión de la velocidad en las vías). El objetivo de los sistemas de transporte inteligente es mejorar la movilidad, seguridad y eficiencia del transporte, mejorando la funcionalidad de los vehículos y las vías usando las tecnologías de la información [4].

\subsection{Proyectos de investigación}

AutoNOMOS, de la Universidad Libre de Berlín, inicia sus trabajos en el año 1998 construyendo robots autónomos, logrando en varias competencias internacionales obtener premios destacados, y el Programa Autopía, de la Universidad Politécnica de Madrid, el cuál fue iniciado en el año 1997, y desde entonces ha centrado su trabajo en la aplicación de técnicas de control, desarrolladas primero para robots móviles, para luego aplicarlas a vehículos autónomos reales.

Programa Autopía El programa Autopía tiene dos objetivos esenciales. El primero, implementar una conducción automática de vehículos comerciales sobre carreteras reales. Aunque este objetivo se puede considerar utópico en este momento, es un punto de partida importante para explorar el futuro. El segundo objetivo es el desarrollo de un sistema de guiado automático formado por componentes modulares que pueda incorporarse de manera sencilla en la industria del automóvil [7].

Las principales entradas sensoriales al sistema de guiado son una cámara de visión y un GPS de alta precisión. El sistema incluye una interfaz de comunicación y un sistema computacional de conducción de bajo nivel en el que reside el conocimiento y la experiencia humana, que se han modelado mediante lógica borrosa [8]. 
AutoNOMOS En la Universidad libre de Berlín, el Dr. Raúl Rojas ha estado construyendo robots autónomos desde 1998. Después de muchas participaciones exitosas en el RoboCup, al ganar el Campeonato Mundial dos veces y el Campeonato Europeo en cinco ocasiones, se decidió extender, como se menciona en [3]. En 2006, se tomó la decisión de participar en Grand Urban Challenge.

Se compró una caravan de Dodge retroadaptada, ya modificada, para que una persona con discapacidad pueda operarla utilizando solo un joystick y un touch pad. Con solo un pequeño presupuesto y 6 meses de tiempo de desarrollo, el auto llamado "Spirit of Berlin"(o más corto, SpoB) llegó a las semifinales de una competencia celebrada por la Agencia de Investigación Avanzada de Defensa (DARPA). Hasta el momento, el proyecto ha funcionado en tres automóviles diferentes [3].

\subsection{Estrategias}

Con el paso del tiempo y los avances tecnológicos, se fueron presentando diferentes mecanismos para el control de velocidad de los vehículos y a continuación se mencionan las siguientes estrategias de implementación.

Acelerador inteligente Con la intención de evitar aceleraciones no intencionadas, algunas marcas utilizan el acelerador inteligente, una solución bastante simple que hace que las órdenes del conductor sobre el pedal del freno siempre prevalezcan sobre las del acelerador. Esto significa, que si se nos queda accionado por error el acelerador, bastaría un pisotón al freno para poder parar el vehículo, ya que si ambos pedales están presionados a la vez la electrónica forzaría al motor a obedecer las órdenes del freno, pudiendo así desacelerar y parar el vehículo [4].

Aplicación android asistente Velociraptor es una nueva aplicación para Android que tiene una función muy especifica pero funciona muy bien. Se trata de un velocímetro que nos indica el límite de velocidad. Al ser una app flotante nos aparecerá en Google Maps. Se basa en nuestra ubicación y en los datos de Here Maps [2].

Asistente de velocidad inteligente (ISA) Dentro del proyecto iSafer, en el año 2017, el servicio de transporte de Londres probó un sistema ISA obligatorio en una pequeña cantidad de autobuses. El sistema demostró ser eficaz y resultó ser especialmente útil para evitar el exceso de velocidad en zonas de $20 \mathrm{mph}$. La prueba halló que, a pesar de que el sistema utilizado era obligatorio, hubo algunas ocasiones en que los autobuses excedieron el límite de velocidad en las secciones de descenso de la carretera. El sistema probado no aplicó automáticamente los frenos, solo evitó la aceleración por encima del límite de velocidad publicado [5]. 


\section{Sistema asistente de velocidad}

En principio se utiliza la librería Javascript de la API de Google Maps. La aplicación es básicamente HTML, CSS y JavaScript trabajando juntos. Los mapas son solo imágenes que se cargan en el fondo a través de peticiones ejecutadas por la tecnología de AJAX, y se insertan en un bloque $<$ div $>$ en la página HTML. Mientras navegas en el mapa, el API envía información acerca de las nuevas coordenadas y los niveles de "zoom" del mapa a través de AJAX y esto retorna las imágenes [9].

\subsection{Modelo del sistema}

Cuando la información de latitud y longitud del GPS se aproxime a los datos que están almacenados en la base de datos, verificará el límite de velocidad almacenado y la velocidad del vehículo. Si se excede el límite de velocidad especificado, el sistema emitirá una alerta al conductor. Teniendo en cuenta el proceso de desarrollo en el cual se encuentra actualmente este trabajo, queremos exponer en la Figura 2 citearticle, el comportamiento dinámico para diferentes valores de $T_{m}$, el cual sería el que más se ajusta a lo que se pretende con este trabajo. En la Figura 1 se ve el diagrama de bloques.

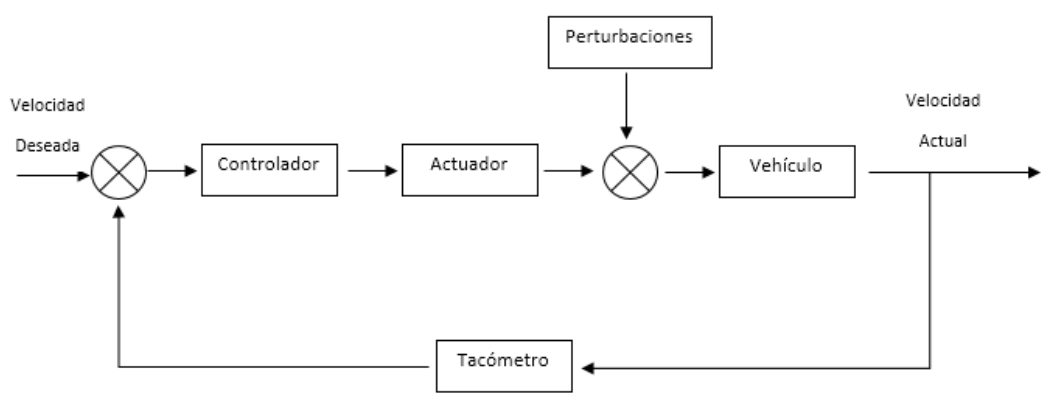

Fig. 1. Sistema de control de velocidad en lazo cerrado.

Requerimientos En cuanto a requerimientos técnicos, el sistema está diseñado para todo tipo de vehículo que cuente con inyección electrónica y con un dispositivo del tipo GPS que permita obtener y evaluar los datos de latitud y longitud.

\section{Ventajas y desventajas}

Ventajas: Control de velocidad en zonas de control por radar, disminución de accidentes, menos emisión de contaminantes al controlar la aceleración.

Desventajas: No reduce velocidad en pendientes, limita su aplicación las especificaciones técnicas de vehículos. 
Rodrigo Velázquez

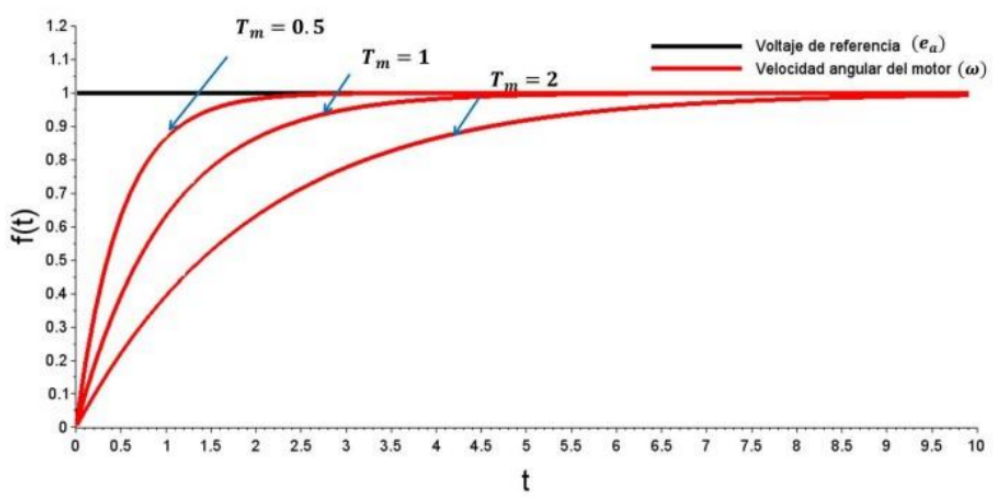

Fig. 2. Respuesta dinámica del modelo del motor de DC para diferentes constantes de tiempo.

\subsection{Aplicación para ingreso de coordenadas}

En esta sección pasaremos a ver las herramientas utilizadas para el desarrollo de la aplicación que permite el ingreso de las coordenadas de las marcas de velocidad. La cual hará posible también que se pueda consultar y comparar con la ubicación actual del vehículo. Como se ve en la Figura 3, la pantalla de ingreso de coordenadas en un teléfono móvil.

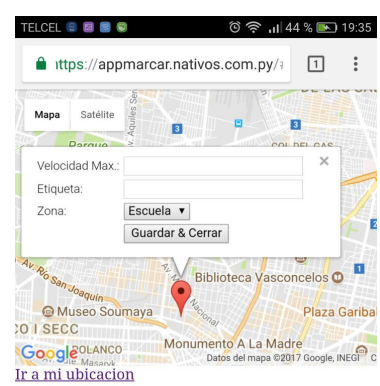

Fig. 3. Esta es la imagen de la aplicación.

Aplicación Las coordenadas están expresadas y almacenadas usando números decimales separados por coma. La latitud siempre precede la longitud. La latitud es positiva si va después del punto mostrado en el mapa y negativo si va antes. En los mapas físicos, las coordenadas están expresadas en grados, así que la posición de La Facultad Politécnica sería:

$$
25^{\circ} 20.7^{\prime} \mathbf{S} 57^{\circ} 31.22^{\prime} \mathbf{O} \text {. }
$$


La forma de convertir estos datos a decimales sería:

- $25^{\circ} 20.7^{\prime}=(25+(20 / 60)+(7 / 3600))=-25.335$,

- $57^{\circ} 31.22^{\prime}=-(57+(31 / 60)+(22 / 3600))=-57.522$.

Como se ve en la Figura 4, así se encuentran almacenadas las coordenadas en la base de datos.

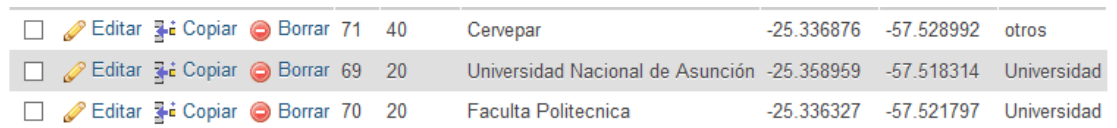

Fig. 4. Ejemplo de cómo se almacenan los datos.

Base de datos Para el almacenamiento de las coordenadas de las marcas de velocidad en las diferentes zonas urbanas se utiliza el SGBD mariaDB. La aplicación mariaDB es un sistema de gestión de bases de datos derivado de MySQL con licencia GPL (General Public License). Es desarrollado por Michael (Monty) Widenius (fundador de MySQL), la fundación mariaDB y la comunidad de desarrolladores de software libre. Introduce dos motores de almacenamiento nuevos, uno llamado Aria -que reemplaza con ventajas a MyISAM- y otro llamado XtraDB -en sustitución de InnoDB. [10]

\section{Conclusiones}

En este trabajo se propuso el modelo que incluye software y hardware, de un sistema asistente de velocidad de un vehículo, según su ubicación en tiempo real, para posteriormente comunicar por medio de sus coordenadas su ubicación con respecto a unas marcas de límite de velocidad y así poder trabajar la información procesada pudiendo utilizarla para alertar al conductor sobre exceso de velocidad. Para su aplicación, el sistema necesitará una conexión a Internet o una base de datos en un dispositivo de almacenamiento dentro del vehículo conectado con GPS.

\section{Referencias}

1. Buenaño-Moyano, L., Mena-Jiménez, R., Venegas-Núñez, J.S., Razo-Sifuentes, A.: Repotenciación de un banco de pruebas de inyección electrónica J20A a través de la adaptación de un sistema de aceleración electrónica TAC, para la implementación en el laboratorio de inyección electrónica de la escuela de ingeniería automotriz (2015)

2. Collado, C.: Ya puedes ver el límite de velocidad de la carretera en google maps gracias a velociraptor (2016) 
3. Czerwionka, P.: A Three Dimensional Map Format for Autonomous Vehicles. Ph.D. thesis, Universidad Libre de Berlín, Instituto de Ciencias de Computación, Alemania

4. Espinoza-Ventura, R.: Sistemas inteligentes de transportes-ITS

5. European Transport Safety Council: iSAFER - ETSC (2017)

6. López-Montes, D.: Sistema de control longitudinal para vehículo eléctrico urbano. Universidad de Cantabria, España (2014)

7. Milanés, V., Naranjo, J., González, C., Alonso, J., García, R., de Pedro, T.: Sistema de posicionamiento para vehículos autónomos. Revista Iberoamericana de Automática e Informática Industrial 5(4), 36-41 (2008)

8. Pérez-Rastelli, J.M.: Agentes de control de vehículos autónomos en entornos urbanos y autovías. Ph.D. thesis, Facultad de Físicas - Universidad Complutense de Madrid, España

9. Rodríguez-Colón, A.: Google Maps API V3 introducción y primeros pasos (2016)

10. Wikipedia: MariaDB (2018) 